\title{
COMBINATORIAL LEMMAS IN HIGHER DIMENSIONS
}

\author{
BY \\ O. BARNDORFF-NIELSEN AND GLEN BAXTER
}

1. Introduction. The starting point for the investigations reported on in this paper was the following result, due to Spitzer and Widom [3], which generalized a similar result due to $\mathrm{Kac}$ [2].

THEOREM 1.1. Let $U=\left\{u_{k} ; k=1,2, \cdots, n\right\}$ be a set of $n$ 2-dimensional vectors and let $s_{k}=u_{1}+\cdots+u_{k}, k=1, \cdots, n$. If $\sigma: i_{1}, i_{2}, \cdots, i_{n}$ is any permutation of $1,2, \cdots, n$, we let $s_{k}(\sigma)=u_{i_{1}}+\cdots+u_{i_{k}}, k=1, \cdots, n$. Finally, let $T_{n}(\sigma)$ denote the length of the boundary of the convex hull $K(\sigma)$ of the path $s_{1}(\sigma), \cdots, s_{n}(\sigma)$ (i.e., the convex hull of the point-set $\left.0, s_{1}(\sigma), s_{2}(\sigma), \cdots, s_{n}(\sigma)\right)$. Then

$$
\sum_{\sigma} T_{n}(\sigma)=2 \sum_{\sigma} \sum_{k=1}^{n} \frac{1}{k}\left|s_{k}(\sigma)\right| \text {. }
$$

If we denote the sum of the vectors in a subset $A$ of $U$ by $u_{A}$ and the number of vectors in $A$ by $n_{A}$, then (1.1).can also be written

$$
\sum_{\sigma} T_{n}(\sigma)=2 \sum_{A}\left(n_{A}-1\right) !\left(n-n_{A}\right) !\left|u_{A}\right| .
$$

Spitzer and Widom derived their result from a formula of Cauchy, expressing the length of the circumference of a compact, convex set in the plane in terms of the lengths of the projections of the set on the lines of the plane and from a lemma of Kac [2, p. 507], asserting the validity of formula (1.1) in the limiting case where the vectors in $U$ all lie on the same line. Thus, in spite of the purely combinatorial nature of this lemma, their proof was not combinatorial. Then one of us (see [1]) succeeded in giving a combinatorial proof of Theorem 1.1 and on the basis of this result the following conjecture was made.

Let $V_{n}(\sigma)$ denote the area of $K(\sigma)$ and let $T\left(u_{A_{1}}, u_{A_{2}}\right)$ denote the area of a triangle with sides $u_{A_{1}}, u_{A_{2}}$ and $u_{A_{1}}+u_{A_{2}}$. Then

$$
\sum_{\sigma} V_{n}(\sigma)=2 \sum_{\sigma}^{\prime}\left(n_{A_{1}}-1\right) !\left(n_{A_{2}}-1\right) !\left(n-n_{A_{1}}-n_{A_{2}}\right) ! T\left(u_{A_{1}}, u_{A_{2}}\right)
$$

where the summation sign $\Sigma^{\prime}$ indicates that we are summing only over nonempty, disjoint subsets $A_{1}$ and $A_{2}$ of $U$.

Received by the editors August 14, 1962.

(1) This research was supported in part by the United States Air Force through the Air Force Office of Scientific Research of the Air Research and Development Command under Contract No. AF 18(603)-30. 
It turned out that the natural way to prove the conjecture was to prove the 3-dimensional analogue of Theorem 1.1, the conjecture being a limiting case thereof. This can be done by generalizing the methods and results in [1] to three dimensions. In fact, propositions of the above-mentioned kind are valid in an arbitrary number of dimensions, as shown in the sequel.

The clue to the whole investigation is the simple observation stated as Lemma 2.1 in $\$ 2$. From this we derive (in $\S 2$ ) two combinatorial lemmas, 2.2 and 2.3, which in turn are used in $\$ 3$ to prove the generalizations of the results of SpitzerWidom and Kac and in $\S 4$ to obtain some formulas of probabilistic interest.

2. Combinatorial lemmas. Let $U=\left\{u_{k} ; k=1, \cdots, n\right\}$ be a set of $n p$-dimensional vectors and let $s_{0}=0, s_{k}=u_{1}+\cdots+u_{k}$. If $\sigma: i_{1}, \cdots, i_{n}$ is any permutation of $1,2, \cdots, n$, we let $s_{k}(\sigma)=u_{i_{1}}+\cdots+u_{i_{k}}$. The notations $u_{A}$ and $n_{A}$ will represent, respectively, the sum of the vectors in a subset $A$ of $U$ and the number of vectors in $A$. The hyperplane determined by $p-1$ linearly independent $p$-dimensional vectors $v_{1}, v_{2}, \cdots, v_{p-1}$ will be denoted by $\gamma\left(v_{1}, \cdots, v_{p-1}\right)$.

Definition 2.1. Let $u_{1}, u_{2}, \cdots, u_{n}$ be $n$-dimensional vectors. We say the set $U=\left\{u_{k} ; k=1, \cdots, n\right\}$ is skew with respect to the hyperplane $\gamma$ of $R^{p}$ if at most one of the vectors $u_{A}, A \subset U$ is contained in $\gamma$.

Our fundamental lemma may now be formulated as follows.

LEMMA 2.1. Let $U=\left\{u_{k} ; k=1, \cdots, n\right\}$ be skew with respect to a $(p-1)$ dimensional hyperplane $\gamma$ containing $s_{n}=u_{U}$. Let $H$ denote one of the halfspaces detrmined by $\gamma$. Then, there exists exactly one cyclic permutation $\sigma$ of $1,2, \cdots, n$ such that exactly $m(m=0,1, \cdots, n-1)$ of the points $s_{1}(\sigma), \cdots, s_{n-1}(\sigma)$ lie in H. (See Figure 2.1 for an example with $m=n-1$.)
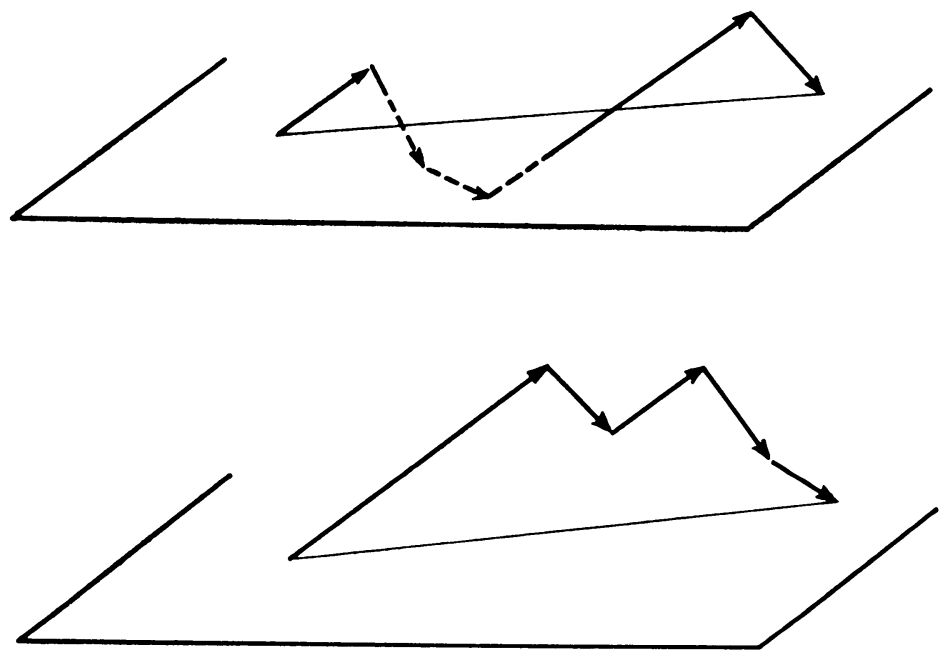

FIGURE 2.1 
Proof. Let $e$ be the unit vector in $H$ perpendicular to $\gamma$. Since $U$ is skew, there exists one and only one index $k$ such that exactly $m$ of the inner products $\left(e, s_{0}\right)$, $\left(e, s_{1}\right), \cdots,\left(e, s_{n-1}\right)$ are greater than $\left(e, s_{k}\right)$. The permutation $\sigma: k+1, \cdots, n, 1, \cdots, k$ has the property stated in the lemma. The uniqueness of $\sigma$ follows from the uniqueness of $k$.

We need another definition.

Definition 2.2. Let $U=\left\{u_{k} ; k=1,2, \cdots, n\right\}$ be a set of $n p$-dimensional vectors $u_{k}$. We say the set is skew if every $p$-tuple of vectors $u_{A_{1}}, u_{A_{2}}, \cdots, u_{A_{p}}$ corresponding to (nonempty) disjoint subsets $A_{1}, \cdots, A_{F}$ of $U$ form a set of linearly independent vectors.

Let $U=\left\{u_{k} ; k=1, \cdots, n\right\}$ be a fixed, skew set of $p$-dimensional vectors and let $\sigma$ be any permutation of $1,2, \cdots, n$. Clearly, any edge in the convex hull of the path

$$
s_{1}(\sigma), s_{2}(\sigma), \cdots, s_{n}(\sigma)
$$

is the sum of the vectors in some subset $A$ of $U$, and this subset is uniquely determined by the edge since $U$ is skew. Now, let $A_{1}, A_{2}, \cdots, A_{r}$ be $r$ disjoint, nonempty subsets of $U, r=p-2$ or $p-1$. Lemma 2.1 enables us to count exactly how many times the whole path

$$
u_{A_{1}}, u_{A_{1}}+u_{A_{2}}, \cdots, u_{A_{1}}+\cdots+u_{A}
$$

lies in the boundary of the convex hull of (2.1), as $\sigma$ ranges over all permutations of $1,2, \cdots, n$, i.e., how many times $u_{A_{1}}, u_{A_{2}}, \cdots, u_{A_{r}}$ are simultaneously edges such that the endpoint of $u_{A_{\rho}}$ coincides with the startpoint of $u_{A_{\rho+1}}, \rho=1,2, \cdots, r-1$. To avoid having to adopt a convention for degenerate cases we assume from now on that $n \geqq p$.

LEMmA 2.2. Let $U=\left\{u_{k} ; k=1, \cdots, n\right\}$ be a fixed, skew set of $n$-dimensional vectors $(p>1)$ and let $A_{1}, \cdots, A_{p-1}$ be fixed, nonempty and disjoint subsets of $U$. Then the whole path

$$
u_{A_{1}}, u_{A_{1}}+u_{A_{2}}, \cdots, u_{A_{1}}+u_{A_{2}}+\cdots+u_{A_{p-1}}
$$

appears in the boundary of the hull of exactly

$$
2\left(n_{A_{1}}-1\right) !\left(n_{A_{2}}-1\right) ! \cdots\left(n_{A_{p-1}}-1\right) !\left(n-n_{A_{1}}-\cdots-n_{A_{p-1}}\right) !
$$

of the $n !$ paths $s_{1}(\sigma), s_{2}(\sigma), \cdots, s_{n}(\sigma)$ as $\sigma$ ranges over all permutations.

Proof. Let $u_{0}=-s_{n}$ and let $A_{0}$ denote the complement of $A_{1} \cup A_{2} \cup \ldots \cup A_{p-1}$ in $U_{0}=\left\{u_{1}, u_{2}, \cdots, u_{n}, u_{0}\right\}$. We call

$$
s_{1}(\sigma), s_{2}(\sigma), \cdots, s_{n}(\sigma), u_{0}
$$


the completed path associated with $u_{i_{1}}, u_{i_{2}}, \cdots, u_{i_{n}}$. Let $H_{1}$ and $H_{2}$ be the two halfspaces in $R$ determined by the hyperplane $\gamma=\gamma\left(u_{A_{1}}, u_{A_{2}}, \cdots, u_{A_{p-1}}\right)$. We include $\gamma$ in both $H_{1}$ and $H_{2}$. We can think of any completed path (2.5) whose hull-boundary contains (2.3) as subdivided naturally into $p$ ordered sets of vectors $A_{1}(\sigma), A_{2}(\sigma), \cdots, A_{p-1}(\sigma)$ and $A_{0}(\sigma), A_{i}(\sigma)$ being the set $A_{i}$ with the ordering induced by $\sigma(i=0,1, \cdots, p-1)$. The paths corresponding to each of these ordered sets of vectors must lie either all in $H_{1}$ or all in $H_{2}$. Moreover, any ordering of the vectors in $A_{1}, \cdots, A_{p-1}$ and $A_{0}$ subject to the condition that their paths lie in the same half-space $H_{j}(j=1,2)$ gives rise to a completed path $s_{1}(\sigma), \cdots, s_{n}(\sigma), u_{0}$ the origin being determined from the position of $u_{0}$ in the ordering of $A_{0}$. Thus we need only to count how many different orderings of vectors in $A_{1}, \cdots, A_{p-1}$ and $A_{0}$ there are such that all $p$ subpaths lie in the same half-space $H_{j}$.

The sets $A_{1}, \cdots, A_{p-1}$ and $A_{0}$ are all skew with respect to $\gamma\left(u_{A_{1}}, \cdots, u_{A_{n-1}}\right)$ since $U$ is skew. Hence, from Lemma 2.1, we find that there are

$$
\left(n_{A_{1}}-1\right) ! \cdots\left(n_{A_{\ldots-1}}-1\right) !\left(n-n_{A_{1}}-\cdots-n_{A_{p-1}}\right) \text { ! }
$$

different ways of ordering $A_{1}, \cdots, A_{p-1}$ and $A_{0}$ such that all $p$ subpaths lie in $H_{1}$, say. Taking into account also $\mathrm{H}_{2}$, the proof is completed.

Let $B_{1}, B_{2}, \cdots, B_{p-1}$ be $p-1$ subsets of $U$. We say the ordered ( $\left.p-1\right)$-tuple $B_{1}, \cdots, B_{p-1}$ has the property $\left({ }^{*}\right)$ (with respect to $A_{1}, \cdots, A_{p-2}$ ) if

$B_{1}, \cdots, B_{p-1}$ are nonempty and disjoint and there exist indices

$v_{1}, v_{2}, \cdots, v_{p-2}$ such that $0 \leqq v_{1}<v_{2}<\cdots<v_{p-2} \leqq p-1$ and

$B_{v_{1}+1} \cup B_{v_{2}}=A_{1}, \quad B_{v_{2}+1} \cup B_{v_{3}}=A_{2}, \cdots, B_{v_{--3}+1} \cup B_{v_{--2}}=A_{p-2}$.

In other words each $A_{k}$ is one of the $B_{k}$ 's, with the possible exception that exactly one of the $A_{k}$ 's is the union of two $B_{k}$ 's.

LEMMA 2.3. Let $U=\left\{u_{k} ; k=1,2, \cdots, n\right\}$ be a fixed, skew set of p-dimensional vectors and let $A_{1}, A_{2}, \cdots, A_{p-2}$ be $p-2$ fixed, nonempty and disjoint subsets of $U$. Then, the whole path

$$
u_{A_{1}}, u_{A_{1}}+u_{A_{2}}, \cdots, u_{A_{1}}+\cdots+u_{A_{p-2}}
$$

lies in the boundary of the hull of exactly

$$
\sum^{*}\left(n_{B_{1}}-1\right) !\left(n_{B_{2}}-1\right) ! \cdots\left(n_{B_{n-1}}-1\right) !\left(n-n_{B_{1}}-\cdots-n_{B_{p-1}}\right) !
$$

of the $n !$ paths $s_{1}(\sigma), \cdots, s_{n}(\sigma)$ as $\sigma$ ranges over all permutations. Here $\sum^{*}$ indicates that we are summing only over ordered $(p-1)$-tuples $B_{1}, \cdots, B_{p-1}$ with the property $\left(^{*}\right)$.

Proof. Let $B_{1}, B_{2}, \cdots, B_{p-1}$ be $p-1$ subsets of $U$ satisfying (*). If the path $u_{B_{1}}, u_{B_{1}}+u_{B_{2}}, \cdots, u_{B_{1}}+\cdots+u_{B_{p-1}}$ appears in the boundary of the hull of $s_{1}(\sigma), \cdots, s_{n}(\sigma)$, then so does (2.6). Conversely, assume that the path (2.6) appears 
in the boundary of the convex hull of $s_{1}(\sigma), \cdots, s_{n}(\sigma)$ and let us consider one of the two sides (of dimension $p-1$ ) of the hull which contains (2.6). Since $U$ is skew, the side contains exactly $p$ vertices of the path $s_{1}(\sigma), \cdots, s_{n}(\sigma)$. Let these vertices be numbered $1,2, \cdots, p$ in accordance with their ordering on the path and let $B_{i}, i=1,2, \cdots, p-1$ denote the subset of $U$ corresponding to the vector from vertex number $i$ to vertex number $i+1$. Then $B_{1}, \cdots, B_{p-1}$ has the property $\left(^{*}\right)$. We may therefore determine the number $n\left(A_{1}, \cdots, A_{p-2}\right)$ of times the path $u_{A_{1}}, u_{A_{1}}+u_{A_{2}}, \cdots, u_{A_{1}}+\cdots+u_{A-2}$ appears in the boundary of the hull of $s_{1}(\sigma), \cdots, s_{n}(\sigma)$ as follows. To any fixed, ordered $(p-1)$-tuple $B_{1}, \cdots, B_{p-1}$ with the property $\left(^{*}\right)$ we count how many times the path $u_{B_{1}}, \cdots, u_{B_{1}}+\cdots+u_{B_{n-1}}$ appears in the boundary of the hull of $s_{1}(\sigma), \cdots, s_{n}(\sigma)$ as $\sigma$ ranges over all permutations. Let us denote this number by $n\left(B_{1}, \cdots, B_{p-1}\right)$. Then

$$
n\left(A_{1}, \cdots, A_{p-2}\right)=\frac{1}{2} \Sigma^{*} n\left(B_{1}, \cdots, B_{p-1}\right)
$$

where $\Sigma^{*}$ indicates that we are summing only over ordered $(p-1)$-tuples $B_{1}, \cdots, B_{p-1}$ satisfying $(*)$.

According to Lemma 2.2 we have

$$
n\left(B_{1}, \cdots, B_{p-1}\right)=2\left(n_{B_{1}}-1\right) ! \cdots\left(n_{B_{n-1}}-1\right) !\left(n-n_{B_{1}}-\cdots-n_{B_{p-1}}\right) ! .
$$

3. Volume and surface area of convex polyhedrons. In this section we derive from Lemma 2.2 a number of combinatorial formulas relating to the volume and surface area of $p$-dimensional convex polyhedrons. Let again $U=\left\{u_{k} ; k=1, \cdots, n\right\}$ be a fixed skew set of $n p$-dimensional vectors and let $\sigma$ be any permutation of $1,2, \cdots, n$. We shall denote the volume and the surface area of the convex hull $K(\sigma)$ of the path $s_{1}(\sigma), s_{2}(\sigma), \cdots, s_{n}(\sigma)$ by $V_{n}(\sigma)$ and $T_{n}(\sigma)$, respectively. The $(p-1)$-dimensional measure of the convex hull of the path $u_{A_{1}}, u_{A_{1}}+u_{A_{2}}, \cdots, u_{A_{1}}+\cdots+u_{A_{n-1}}$ where $A_{p}, \cdots, A_{p-1}$ is any set of $p-1$ nonempty and disjoint subsets of $U$, will be denoted by $T\left(u_{A_{1}}, u_{A_{2}}, \cdots, u_{A_{-1}}\right)$.

In order to clarify the exposition we have chosen to discuss the cases $p=1,2$ and 3 before passing over to the general results.

Let $p=2$. Then $T_{n}(\sigma)$ is the length of the boundary of $K(\sigma)$ and we have the following formula due to Spitzer and Widom:

$$
\sum_{\sigma} T_{n}(\sigma)=2 \sum_{A}\left(n_{A}-1\right) !\left(n-n_{A}\right) !\left|u_{A}\right|,
$$

where $\left|u_{A}\right|$ is the length of the vector $u_{A}$.

As was pointed out in [1], this relation may be proved as follows. By definition, $T_{n}(\sigma)$ equals the sum of the lengths of the edges in $K(\sigma)$. It was mentioned in $\$ 2$ that each edge in $K(\sigma)$ is the sum of the vectors in a uniquely determined subset $A$ of $U$. According to Lemma 2.2, the vector $u_{A}$ appears as an edge in exactly 
$2\left(n_{A}-1\right) !\left(n-n_{A}\right)$ ! of the convex hulls $K(\sigma)$ as $\sigma$ ranges over all permutations. This proves the formula (3.1).

Next, let $p=3$. In this case, $T_{n}(\sigma)$ equals the surface area of the 3-dimensional polyhedron $K(\sigma), T\left(u_{A_{1}}, u_{A_{2}}\right)$ is the area of a triangle with sides $u_{A_{1}}, u_{A_{2}}$, and $u_{A_{1}}+u_{A_{2}}$, and

$$
\sum_{\sigma} T_{n}(\sigma)=2 \Sigma^{\prime}\left(n_{A_{1}}-1\right) !\left(n_{A_{2}}-1\right) !\left(n-n_{A_{1}}-n_{A_{2}}\right) ! T\left(u_{A_{1}}, u_{A_{2}}\right)
$$

where the summation sign $\Sigma^{\prime}$ indicates that we are summing only over ordered pairs of nonempty, disjoint subsets $A_{1}$ and $A_{2}$ of $U$. The proof of this relation is completely analogous to that of (3.1). Formula (3.2) can be reduced to a form more in line with the formulas of Kac [2] and Spitzer-Widom [3]:

$$
\sum_{\sigma} T_{n}(\sigma)=2 \sum_{\sigma} \sum_{k, m=1}^{n} \frac{1}{k m} \hat{T}\left(s_{k}(\sigma), s_{m}(\sigma)\right),
$$

where $\hat{T}\left(s_{k}(\sigma), s_{m}(\sigma)\right)$ is the area of the triangle with vertices at $0, s_{k}(\sigma), s_{m}(\sigma)$. The general theorem is as follows.

THEOREM 3.1. Let $U=\left\{u_{k} ; k=1, \cdots, n\right\}$ be a fixed, skew set of p-dimensional vectors, let $T_{n}(\sigma)$ be the surface area of the convex hull of the path $s_{1}(\sigma), s_{2}(\sigma), \cdots, s_{n}(\sigma)$ and let $T\left(u_{A_{1}}, \cdots, u_{A_{n-1}}\right)$ be the $(p-1)$-dimensional measure of the convex hull of the path $u_{A_{1}}, u_{A_{1}}+u_{A_{2}}, \cdots, u_{A_{1}}+\cdots+u_{A_{-1}}$. Then

$$
\begin{aligned}
\sum_{\sigma} & T_{n}(\sigma) \\
\quad & =2 \Sigma^{\prime}\left(n_{A_{1}}-1\right) ! \cdots\left(n_{A_{p-1}}-1\right) !\left(n-n_{A_{1}}-\cdots-n_{A_{p-1}}\right) ! T\left(u_{A_{1}}, \cdots, u_{A_{n-1}}\right)
\end{aligned}
$$

where the summation sign $\Sigma^{\prime}$ indicates that we are summing only over ordered ( $p-1)$-tuples of nonempty, disjoint subsets $A_{1}, A_{2}, \cdots, A_{p-1}$ of $U$.

Proof. The left side in (3.3) equals the sum of the areas with ( $p-1)$-dimensional measures of the sides in the convex hulls $K(\sigma)$, where $\sigma$ ranges over all permutations. Each side in a given $K(\sigma)$ is the convex hull of one and only one subpath $u_{A_{1}}, u_{A_{1}}+u_{A_{2}}, \cdots, u_{A_{1}}+\cdots+u_{A_{p-1}}$ of $s_{1}(\sigma), s_{2}(\sigma), \cdots, s_{n}(\sigma)$, where $A_{1}, A_{2}, \cdots, A_{p-1}$ are nonempty and disjoint. Lemma 2.2 tells us that this particular subpath will appear in the boundary of exactly

$$
2\left(n_{A_{1}}-1\right) ! \cdots\left(n_{A_{p-1}}-1\right) !\left(n-n_{A_{1}}-\cdots-n_{A_{n-1}}\right) \text { ! }
$$

of the hulls $K(\sigma)$ as $\sigma$ ranges over all permutations. This completes the proof.

Let $p=1$. In their proof of formula (3.1), Spitzer and Widom exploited a result of $\mathrm{M}$. Kac [2] to the effect that

$$
\sum_{\sigma}\left(\max _{0 \leqq k \leqq n} s_{k}(\sigma)-\min _{0 \leqq k \leqq n} s_{k}(\sigma)\right)=\sum_{\sigma} \sum_{k=1}^{n} \frac{1}{k}\left|s_{k}(\sigma)\right| .
$$


Formula (3.4) is in fact a limiting case of (3.1). To see this, we note that the right hand side of (3.4) may be written as

$$
\sum_{\sigma}\left(\max _{0 \leqq k \leqq n} s_{k}(\sigma)-\min _{0 \leqq k \leqq n} s_{k}(\sigma)\right)=\sum_{A}\left(n_{A}-1\right) !\left(n-n_{A}\right) !\left|u_{A}\right| .
$$

To any set $U=\left\{u_{k} ; k=1, \cdots, n\right\}$ of (nondegenerate) 1-dimensional vectors we may find a skew set $Z=\left\{z_{k} ; k=1, \cdots, n\right\}$ of 2-dimensional vectors $z_{k}$ such that the projection of $z_{k}$ on the line containing the vectors in $U$ is equal to $u_{k}$, $k=1, \cdots, n$. We may actually find a whole sequence $Z^{(1)}, Z^{(2)}, \cdots, Z^{(i)}, \cdots$ of sets $Z^{(i)}=\left\{z_{k}^{(i)} ; k=1, \cdots, n\right\}$ of the above-mentioned type such that $z_{k}^{(i)} \rightarrow u_{k}$ as $i \rightarrow \infty(k=1, \cdots, n)$. For each set $Z^{(i)}$, a formula like (3.1) holds; in symbols

$$
\sum_{\sigma} T_{n}^{(i)}(\sigma)=2 \sum_{A}\left(n_{A}-1\right) !\left(n-n_{A}\right) ! T\left(z_{A}^{(i)}\right) .
$$

As $i$ approaches infinity we have

$$
T_{n}^{(i)}(\sigma) \rightarrow 2\left(\max _{0 \leqq k \leqq n} s_{k}(\sigma)-\min _{0 \leqq k \leqq n} s_{k}(\sigma)\right)
$$

and

$$
T\left(z_{\boldsymbol{A}}^{(i)}\right) \rightarrow T\left(u_{A}\right)=\left|u_{A}\right|
$$

and this verifies (3.5) (and hence (3.4)).

Now, let $p=2$. We have the following 2-dimensional analogue of formula

$$
\sum_{\sigma} V_{n}(\sigma)=\Sigma^{\prime}\left(n_{A_{1}}-1\right) !\left(n_{A_{2}}-1\right) !\left(n-n_{A_{1}}-n_{A_{2}}\right) ! T\left(u_{A_{1}}, u_{A_{2}}\right)
$$

where, by definition, $V_{n}(\sigma)$ is the area of the convex hull of $s_{1}(\sigma), s_{2}(\sigma), \cdots, s_{n}(\sigma)$. (The summation sign $\Sigma^{\prime}$ indicates the sum over all ordered and nonempty, disjoint subsets $A_{1}$ and $A_{2}$ of $U$.) In fact, somewhat more is true. Suppose that to every given ordered pair $A_{1}, A_{2}$ of nonempty, disjoint subsets of $U$, we have $\left(n_{A_{1}}-1\right) !\left(n_{A_{2}}-1\right) !\left(n-n_{A_{1}}-n_{A_{2}}\right)$ ! triangles with sides $u_{A_{1}}$ and $u_{A_{2}}$. It is then possible to arrange all these triangles into $n$ ! groups, each group corresponding to one of the $n$ ! permutations $\sigma$, in such a way that within each group the triangles may be fitted together so as to cover exactly the convex hull of the path determined by the permutation which characterizes the group.

If, for instance, the set $U$ consists of the four vectors below, and if we have

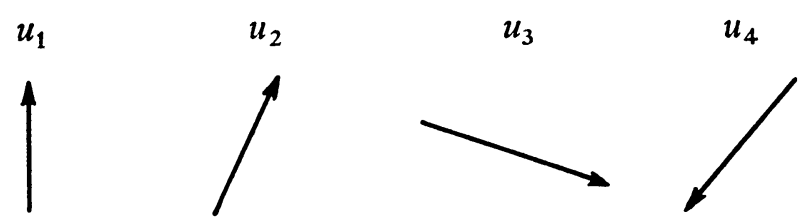

Figure 3.1 
four triangles with sides $u_{1}$ and $u_{2}$ (two corresponding to the pair $u_{1}, u_{2}$ and two to the pair $u_{2}, u_{1}$ ), four with sides $u_{1}$ and $u_{3}$, etc., two triangles with sides $u_{1}$ and $u_{2}+u_{3}$, etc., then these may be fitted together in a (by no means unique) way, so as to cover exactly and at the same time all the $n !=24$ convex hulls generated by $u_{1}, \cdots, u_{4}$. An example of such a covering is shown in Figure 3.2, where the triangles have been numbered with congruent triangles having the same number. Table 3.1 reveals which vectors determine a given triangle.
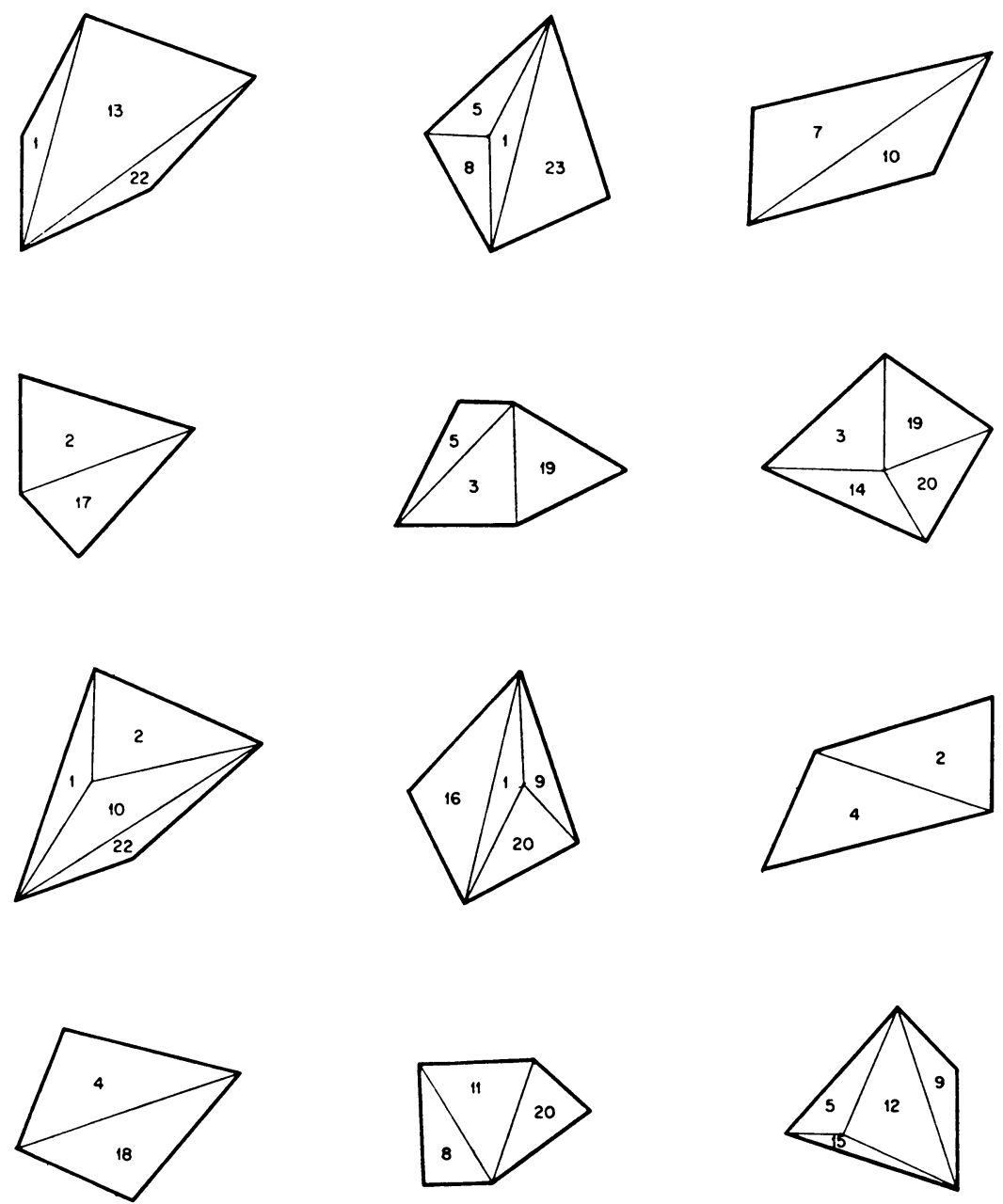

FIGURE 3.2a 

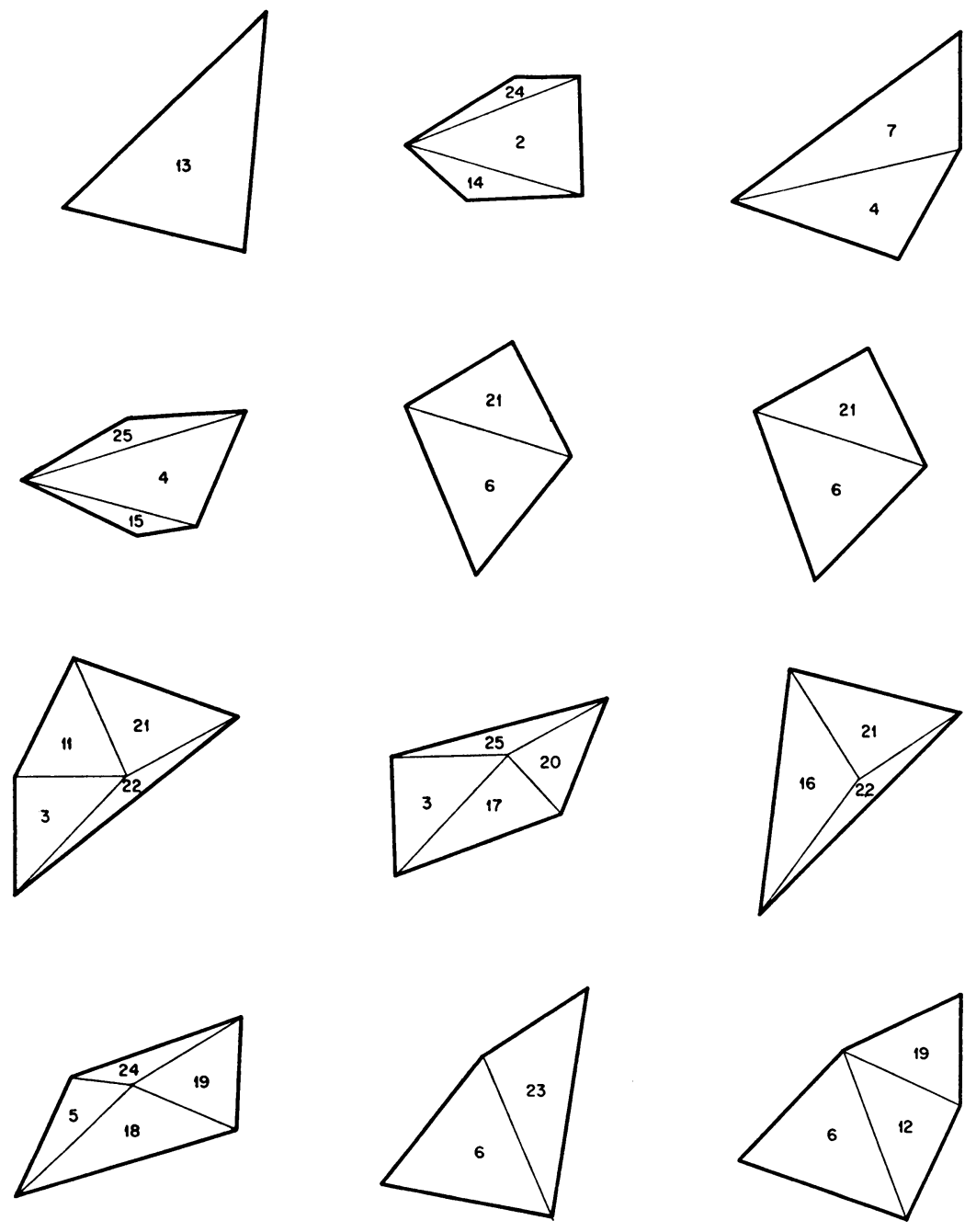

FIGURE 3.2b

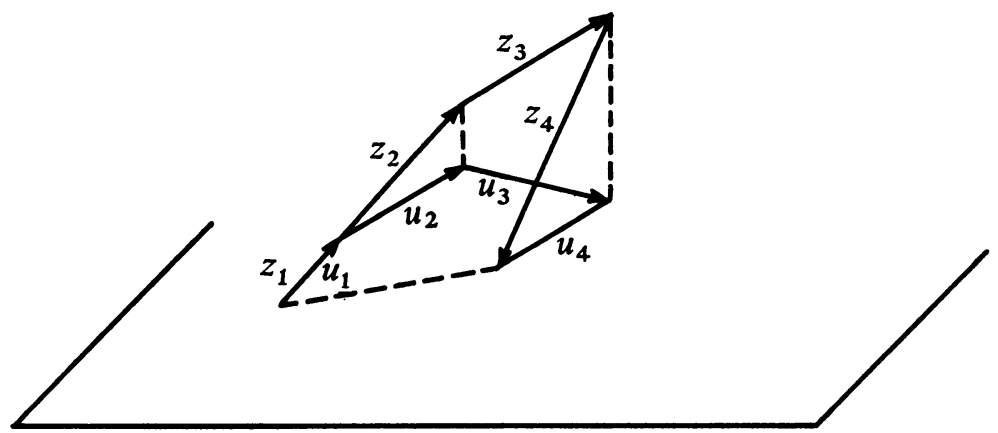

FIGURE 3.3 
TABLE 3.1

$\begin{array}{clcc}\begin{array}{c}\text { triangle } \\ \text { no. }\end{array} & \begin{array}{c}\text { determined } \\ \text { by }\end{array} & \begin{array}{c}\text { triangle } \\ \text { no. }\end{array} & \begin{array}{c}\text { determined } \\ \text { by }\end{array} \\ 1 & u_{1}, u_{2} & 14 & u_{3}, u_{1}+u_{4} \\ 2 & u_{1}, u_{3} & 15 & u_{3}, u_{2}+u_{4} \\ 3 & u_{1}, u_{4} & 16 & u_{4}, u_{1}+u_{2} \\ 4 & u_{2}, u_{3} & 17 & u_{4}, u_{1}+u_{3} \\ 5 & u_{2}, u_{4} & 18 & u_{4}, u_{2}+u_{3} \\ 6 & u_{3}, u_{4} & 19 & u_{1}, u_{2}+u_{3}+u_{4} \\ 7 & u_{1}, u_{2}+u_{3} & 20 & u_{2}, u_{1}+u_{3}+u_{4} \\ 8 & u_{1}, u_{2}+u_{4} & 21 & u_{3}, u_{1}+u_{2}+u_{4} \\ 9 & u_{1}, u_{3}+u_{4} & 22 & u_{4}, u_{1}+u_{2}+u_{3} \\ 10 & u_{2}, u_{1}+u_{3} & 23 & u_{1}+u_{2}, u_{3}+u_{4} \\ 11 & u_{2}, u_{1}+u_{4} & 24 & u_{1}+u_{3}, u_{2}+u_{4} \\ 12 & u_{2}, u_{3}+u_{4} & 25 & u_{1}+u_{4}, u_{2}+u_{3} \\ 13 & u_{3}, u_{1}+u_{2} & & \end{array}$

To see this, let us consider a skew set $Z$ of 4 3-dimensional vectors $z_{1}, z_{2}, z_{3}$ and $z_{4}$ such that $z_{k}$ projects into $u_{k}, k=1, \cdots, 4$. In our example the $z$-vectors have the coordinates $z_{1}=(0,2,0), z_{2}=(1,2,1), z_{3}=(3,-1,2)$ and $z_{4}=$ $(-2,-2,-3)$ (see Figure 3.3). Let $e$ be a unit vector perpendicular to the plane $\gamma$ spanned by $u_{1}, \cdots, u_{4}$ and let $K_{0}(\sigma), \sigma: i_{1}, i_{2}, i_{3}, i_{4}$, be the convex hull of the path $z_{i_{1}}, z_{i_{1}}+z_{i_{2}}, z_{i_{1}}+z_{i_{2}}+z_{i_{3}}, z_{i_{1}}+z_{i_{2}}+z_{i_{3}}+z_{i_{4}}$. We provide each of the triangular sides of $K_{0}(\sigma)$ with a unit vector $f$ perpendicular to the side and pointing away from $K_{0}(\sigma)$. Let us call a side with unit vector $f$ red if the inner product $(e, f)$ is positive and white if $(e, f)$ is negative $((e, f)$ cannot be a zero since $Z$ is skew). The red triangles project onto $\gamma$ into (nonoverlapping) triangles fitting together so as to cover exactly the convex hull of $u_{i_{1}}, \cdots, u_{i_{1}}+\cdots+u_{i_{4}}$. Each of the projected triangles is the convex hull of one and only one subpath $u_{A_{1}}, u_{A_{1}}+u_{A_{2}}$ of $u_{i_{1}}, \cdots, u_{i_{1}}+\cdots+u_{i_{4}}$ and is the projection of the convex hull of the uniquely determined subpath of $z_{i_{1}}, \cdots, z_{i_{1}}+\cdots+z_{i_{4}}$ which projects into $u_{A_{1}}, u_{A_{1}}+u_{A_{2}}$. According to Lemma 2.2 this subpath of $z_{i_{1}}, \cdots, z_{i_{1}}+\cdots+z_{i_{4}}$ lies in the boundary of exactly $2\left(n_{A_{1}}-1\right) !\left(n_{A_{2}}-1\right) !\left(n-n_{A_{1}}-n_{A_{2}}\right)$ ! of the convex hulls $K_{0}(\sigma)$, there determining either a red or a white side of $K_{0}(\sigma)$. As may be seen from the proof of that lemma, the subpath must give rise to equally many red and white sides. This proves the assertion.

Note that the decomposition of the hulls into triangles as described above is not unique. There are several possible decompositions depending on the values of the third components of the vectors $z_{1}, \cdots, z_{4}$. The appearance of a given triangle in one of the hulls indicates that a certain set of inequalities is satisfied. If the third components are altered, the inequalities may be reversed, in which case a new decomposition will arise. Nonetheless, the total set of triangles remains unaltered. 
We now formulate the general result, which may be proved by a straightforward modification of the argument given above for the case $p=2$ and $n=4$.

THEOREM 3.2. Let $U=\left\{u_{k} ; k=1, \cdots, n\right\}$ be a fixed, skew set of $p$-dimensional vectors. Suppose that to every given ordered set $A_{1}, A_{2}, \cdots, A_{p}$ of $p$ nonempty, disjoint subsets of $U$, we have

$$
\left(n_{A_{1}}-1\right) !\left(n_{A_{2}}-1\right) ! \cdots\left(n_{A_{n}}-1\right) !\left(n-n_{A_{1}}-\cdots-n_{A_{n}}\right) !
$$

copies of the convex hull of the path $u_{A_{1}}, u_{A_{1}}+u_{A_{2}}, \cdots, u_{A_{1}}+\cdots+u_{A_{n}}$. It is then possible to arrange all these convex polyhedrons into $n !$ groups in at least one way such that there is a one-to-one correspondence between the groups and the $n$ ! permutations and such that within each group the polyhedrons may be fitted together so as to yield the convex hull of the path $s_{1}(\sigma), s_{2}(\sigma), \cdots, s_{n}(\sigma)$ determined by the permutation which characterizes the group.

Denoting the volume of the convex hull of the path $s_{1}(\sigma), \cdots, s_{n}(\sigma)$ by $V_{n}(\sigma)$ and the volume of the convex hull of the path $u_{A_{1}}, \cdots, u_{A_{1}}+\cdots+u_{A_{n}}$ by $T\left(u_{A_{1}}, \cdots, u_{A_{r}}\right)$ we consequently have

$$
\sum_{\sigma} V_{n}(\sigma)=\Sigma^{\prime}\left(n_{A_{1}}-1\right) ! \cdots\left(n_{A_{n}}-1\right) !\left(n-n_{A_{1}}-\cdots-n_{A_{n}}\right) ! T\left(u_{A_{1}}, \cdots, u_{A_{p}}\right)
$$

where the summation sign $\Sigma^{\prime}$ indicates that we are summing only over nonempty, disjoint subsets $A_{1}, A_{2}, \cdots, A_{p}$ of $U$. Theorem 3.2 remains true even if $U$ $=\left\{u_{k} ; k=1, \cdots, n\right\}$ is not skew.

4. Applications to probability theory. The formulas given in $\S \S 2$ and 3 may be used to obtain results of probabilistic interest. We illustrate this by some examples.

Let $X_{k}=\left(X_{k}^{(1)}, \cdots, X_{k}^{(p)}\right) ; k=1, \cdots, n$, be a set of $n$ independent and identically distributed $p$-dimensional random variables. We assume for convenience that $X_{k}^{(1)}, \cdots, X_{k}^{(p)}$ have a joint density function; this implies that with probability one $X_{1}, \cdots, X_{n}$ is skew. If $\sigma: i_{1}, \cdots, i_{n}$ is any permutation of $1, \cdots, n$, we let $S_{0}(\sigma)=0, S_{1}(\sigma)=X_{i_{1}}, \cdots, S_{n}(\sigma)=X_{i_{1}}+\cdots+X_{i_{n}}$ and we denote the convex hull of the path $S_{1}(\sigma), \cdots, S_{n}(\sigma)$ by $K(\sigma)$. We shall indicate the identity permutation $\sigma: 1,2, \cdots, n$ by deleting the symbol $(\sigma)$ from our notations. The expectation of a random variable $X$ will be denoted by $E\{X\}$.

EXAMPLE 4.1. Let $H_{n}(\sigma)$ be the number of sides in $K(\sigma)$. Then, by the identical distribution property we have $E\left\{H_{n}\right\}=E\left\{H_{n}(\sigma)\right\}$ for any permutation $\sigma$ and hence

$$
E\left\{H_{n}\right\}=\frac{1}{n !} E\left\{\sum_{\sigma} H_{n}(\sigma)\right\}
$$


The summation on the right in (4.1) equals the total number of sides in the $n$ ! hulls $K(\sigma)$ as $\sigma$ ranges over all permutations.

Thus, if $p=2$, we have according to Lemma 2.2 (with probability one)

$$
\begin{aligned}
\sum_{\sigma} H_{n}(\sigma) & =2 \sum_{A}\left(n_{A}-1\right) !\left(n-n_{A}\right) ! \\
& =2 \sum_{k=1}^{n}\left(\begin{array}{l}
n \\
k
\end{array}\right)(k-1) !(n-k) ! \\
& =2 n ! \sum_{k=1}^{n} \frac{1}{k} .
\end{aligned}
$$

Hence

$$
E\left\{H_{n}\right\}=2 \sum_{k=1}^{n} \frac{1}{k} \sim \angle \log n .
$$

Similarly, if $p=3$, we find from Lemma 2.2

$$
E\left\{H_{n}\right\}=2 \sum_{k=1}^{n-1} \sum_{m=1}^{n-k} \frac{1}{k \cdot m} \sim 2(\log n)^{2} .
$$

EXAmple 4.2. Let $p=3$ and let $I_{n}(\sigma)$ denote the number of edges in $K(\sigma)$. Then

$$
E\left\{I_{n}\right\}=3 \sum_{k=1}^{n-1} \sum_{m=1}^{n-k} \frac{1}{k \cdot m} \sim 3(\log n)^{2}
$$

This result may be derived from Lemma 2.3 by a reasoning similar to that given in Example 4.1. It follows, however, immediately from formula (4.4) and the fact that, if $X_{1}, \cdots, X_{n}$ is skew, then for every $\sigma$ the sides in $K(\sigma)$ are triangles and hence we must have $I_{n}(\sigma)=(3 / 2) H_{n}(\sigma)$ and consequently $E\left\{I_{n}\right\}=(3 / 2) E\left\{H_{n}\right\}$.

EXAmple 4.3. Let $p=2$. From Theorem 3.1 we find the following formula (due to Spitzer and Widom [3]) for the expectation of the length $T_{n}$ of the boundary of $K$

$$
\begin{aligned}
E\left\{T_{n}\right\} & =\frac{1}{n !} E\left\{\sum_{\sigma} T_{n}(\sigma)\right\}=\frac{2}{n !} \sum_{A}\left(n_{A}-1\right) !\left(n-n_{A}\right) ! E\left\{T\left(X_{A}\right)\right\} \\
& =\frac{2}{n !} \sum_{k=1}^{n}\left(\begin{array}{l}
n \\
k
\end{array}\right)(k-1) !(n-k) ! E\left\{T\left(S_{k}\right)\right\} \\
& =2 \sum_{k=1}^{n} \frac{1}{k} E\left|S_{k}\right| .
\end{aligned}
$$

In the case $p=3$ we have 


$$
\begin{aligned}
E\left(T_{n}\right)= & \frac{2}{n !} \sum^{\prime}\left(n_{A_{1}}-1\right) !\left(n_{A_{2}}-1\right) !\left(n-n_{A_{1}}-n_{A_{2}}\right) ! E\left\{T\left(X_{A_{1}}, X_{A_{2}}\right)\right\} \\
= & \frac{2}{n !} \sum_{k=1}^{n-1} \sum_{m=1}^{n-k} \frac{n !}{k ! m !(n-k-m) !}(k-1) !(m-1) !(n-k-m) ! \\
= & 2 \sum_{k=2}^{n} \sum_{m=1}^{k-1} \frac{1}{m(k-m)} E\left\{T\left(S_{m}, S_{k}-S_{m}\right)\right\} .
\end{aligned}
$$

EXAMPLE 4.4. As a last example we consider for $p=2$ the area $V_{n}$ of the convex hull $K$. From formula (3.10) we obtain in the same manner as above

$$
E\left(V_{n}\right)=\sum_{k=2}^{n} \sum_{m=1}^{k-1} \frac{1}{m(k-m)} E\left\{T\left(S_{m}, S_{k}-S_{m}\right)\right\}
$$

If, in particular, the components $X_{k}^{(1)}$ and $X_{k}^{(2)}$ of $X_{k}$ have expectation 0 and finite second moment, then $n^{-1 / 2} S_{n}$ has a bivariate normal limiting distribution and $\left|n^{-1 / 2} S_{n}\right|$ is uniformly integrable in $n$. Thus

$$
\begin{aligned}
m^{-1 / 2}(k-m)^{-1 / 2} E\left\{T\left(S_{m}, S_{k}-S_{m}\right)\right\} & =E\left\{T\left(m^{-1 / 2} S_{m},(k-m)^{-1 / 2}\left(S_{k}-S_{m}\right)\right\}\right. \\
& \rightarrow c \quad \text { as }\left\{\begin{array}{l}
m \rightarrow \infty, \\
k-m \rightarrow \infty,
\end{array}\right.
\end{aligned}
$$

where $c$ is a finite constant. In the case of rotational symmetry of the limiting distribution it is easy to see that $2 c=E\left\{X_{k}^{(1)^{2}}\right\}=E\left\{X_{k}^{(2)^{2}}\right\}$. It follows, by elementary calculations, that

$$
\frac{E\left\{V_{n}\right\}}{n} \rightarrow c \pi \quad \text { as } \quad n \rightarrow \infty
$$

\section{REFERENCES}

1. G. Baxter, A combinatorial lemma for complex numbers, Ann. Math. Statist. 32 (1961), 901-904.

2. M. Kac, Toeplitz matrices, translation kernels, and a related problem in probability theory, Duke Math. J. 21 (1954), 501-509.

3. F. Spitzer and H. Widom, The circumference of a convex polygon, Proc. Amer. Math. Soc. 12 (1961), 506-509.

AARHUS UNIVERSITET,

AARHUS, DENMARK

UNIVERSITY OF MINNESOTA,

MINNEA POLIS, MiNNESOTA 